\title{
Une Méthode de Dosage Radioimmunologique du Glucagon comportant une Séparation par le Charbon-Dextran
}

\author{
V. Leclercq-Meyer, P. Mialhe et W.J. Malaisse \\ Institut de Physiologie et de Chimie Biologique, Université de Strasbourg, France (Prof. F. Stutinsky) et Laboratoire de \\ Médecine Expérimentale, Université Libre de Bruxelles, Balgique (Prof. P.A. Bastenie)
}

\author{
Reçu le 8 Octobre 1969
}

\begin{abstract}
Dextran-coated charcoal radioimmunoassay of glucagon
Summary. A dextran-coated charcoal radioimmuno. assay of glucagon is described. Antigen ( ${ }^{131}$ or ${ }^{125}$ I-glucagon, $0.060 \mathrm{ng}$ ) - antibody (rabbit antiserum 1:550, final dilution 1:6600) reactions reached equilibrium within 3 days at $4^{\circ} \mathrm{C}$. The use of a proteinase inhibitor (Trasylol) prevented incubation damage of the immunoreactive glucagon. The sensitivity of the assay ( $<$ to $0.020 \mathrm{ng}$ ) corresponds to about $0.100 \mathrm{ng} / \mathrm{ml}$ of plasma. The precision, the reproducibility, the specificity of the assay and some problems related to the dextran-charcoal separation have been studied. Recovery of glucagon added to plasma was approximatively $90 \%$. The mean glucagon concentration measured in peripheral venous plasma was 0.208 $\mathrm{ng} / \mathrm{ml}$ in normal human subjects after an overnight fast, $0.396 \mathrm{ng} / \mathrm{ml}$ in anaesthetized dogs after an overnight fast, $0.354 \mathrm{ng} / \mathrm{ml}$ in fed rats and $0.909 \mathrm{ng} / \mathrm{ml}$ in the overnightfasted duck. Up to now, the use of the assay seems to be restricted to studies of glucagon secretion in vitro since our antiserum cross-reacts with a material extracted from the duodenum. The relative contribution of this material in the plasma glucagon determinations remains, however, to be established.
\end{abstract}

Résumé. Une méthode de dosage radioimmunologique du glucagon comportant une séparation de l'antigène marqué libre et de l'antigène marqué lié à l'anticorps par du charbon-dextran est décrite. Les réactions d'équilibre glucagon-131 I ou ${ }^{-125} \mathrm{I}(0.060 \mathrm{ng}$ ) et anticorps (antisérum de lapin 1:550, dilution finale 1:6600) atteignent leur équilibre en 3 jours à $4^{\circ} \mathrm{C}$. L'emploi d'un inhibiteur des protéinases (Trasylol) empêche la dégradation de la fraction immunoréactive du glucagon pendant l'incubation. La sensibilité de la méthode (< à $0.020 \mathrm{ng}$ ) correspond à environ $0.100 \mathrm{ng} / \mathrm{ml}$ de plasma. La précision, la reproductibilité, la spécificité du dosage et quelques problèmes liés à la séparation par le charbon-dextran sont étudiés. La récupération du glucagon ajouté à du plasma est d'environ $90 \%$. La concentration en glucagon dans le plasma veineux périphérique est en moyenne de 0.208 $\mathrm{ng} / \mathrm{ml}$ chez l'homme normal à jeun, de $0.396 \mathrm{ng} / \mathrm{ml} \mathrm{chez}$ lo chien anesthésié à jeun, de $0.354 \mathrm{ng} / \mathrm{ml}$ chez le rat nourri et de $0.909 \mathrm{ng} / \mathrm{ml}$ chez le canard à jeun. La méthode de dosage proposée semble actuellement limitée à l'étude de la sécrétion du glueagon in vitro car l'antisérum que nous utilisons présente une réaction croisée avec une substance d'origine duodénale. L'importance de l'interférence de cette substance dans l'estimation des concentrations en glucagon du plasma reste cependant à déterminer.

Eine radioimmunologische Bestimmungsmethode für Glucagon mit Hilfe einer Trennung durch Dextran-Holzkohle

Zusammenfassung. Eine radioimmunologische Bestimmungsmethode für Glucagon unter Zuhilfenahme von Dextran-Holzkohle für die Trennung des freien und des antikörpergebundenen markierten Antigens wird beschrieben. Das Gleichgewicht zwischen mit ${ }^{125} \mathrm{I}$ und ${ }^{131} \mathrm{I}$ markiertem Glucagon (0.060 ng) und Antikörper (Kaninchen-Antiserum 1:550 in einer Endverdünnung von $1: 6600$ ) wird bei $4^{\circ} \mathrm{C}$ in 3 Tagen erreicht. Der Zusatz eines Proteinasen-Hemmers (Trasylol) verhindert den Abbau der immunreaktiven Fraktion des Glucagons während der Inkubation. Die Empfindlichkeit der Methode erreicht etwa $0.100 \mathrm{ng} / \mathrm{ml}$ Plasma. Die Genauigkeit, die Reproduzierbarkeit, die Spezifität und einige Probleme, die bei der Trennung durch Dextran-Holzkohle auftauchen, werden untersucht. Die Wiederauffindungsquote für dem Plasma zugesetztes Glucagon betrug ca. 90\%. Die mittlere Glucagon-Konzentration im peripheren venösen Plasma bei fastenden Normalpersonen lag bei $0.208 \mathrm{ng} / \mathrm{ml}$, beim fastenden anaesthesierten Hund bei $0.396 \mathrm{ng} / \mathrm{ml}$, bei $0.354 \mathrm{ng} / \mathrm{ml}$ für die gefütterte Ratte und bei $0.909 \mathrm{ng} / \mathrm{ml}$ bei fastenden Enten. Die vorgeschlagene Bestimmungsmethode scheint nur für Untersuchungen über die in vitro Sektretion von Glucagon brauchbar zu sein, da das von uns verwandte Antiserum eine Kreuzreaktion mit einer aus dem Duodenum stammenden Substanz zeigt. Wie stark dieser Stoff die Messung von Glucagon-Konzentrationen im Plasma stört, bleibt noch festzustellen.

Key-words: Radioimmunoassay of glucagon, glucagon secretion.
Au cours des 10 dernières années, plusieurs groupes d'auteurs ont décrit des méthodes de dosage radioimmunologiques du glucagon. La séparation de l'antigène marqué libre et de l'antigène marqué lié à l'anticorps est effectuée par chromatoélectrophorèse sur papier $[22,6,10,2]$, précipitation par un second anticorps [7], adsorption sur amberlite G-400 [23] ou sur poudre de cellulose [13]. L'application de ces méthodes a déjà apporté de nombreuses informations en ce qui concerne la physiologie du glucagon, mais des contradictions majeures subsistent et justifient l'intérêt de nouvelles recherches en ce domaine. C'est pourquoi nous nous proposons de décrire et d'analyser ici une méthode de dosage radioimmunologique du glucagon comportant une séparation par du charbon-dextran. Des résultats préliminaires obtenus avec cette méthode sont également présentés. 


\section{Matériel et méthodes}

La séparation du glucagon marqué libre et du glucagon marqué lié à l'anticorps est effectuée, après une incubation classique, par une suspension de charbon-dextran. Cette technique est une modification de celle qui a été proposée pour le dosage radioimmunologique de l'insuline [9] et que nous avons déjà employée pour le dosage de l'hormone somatotrope chez le singe rhesus [11].

\section{Glucagons utilisés}

Le glucagon que nous utilisons pour les immunisations, la préparation des standards de la courbe de référence et les études de récupération de l'hormone dans le plasma est du glucagon de boeuf-pore Lilly (Lot $\mathbf{n}^{\circ}$ 258-234-B-167-1).

Le glucagon marqué utilisé comme traceur est soit du glucagon- ${ }^{131} \mathrm{I}$ avec des radioactivités spécifiques variant entre 320 et $880 \mu \mathrm{Ci} / \mu \mathrm{g}$ [14] (glucagon de porc Novo, Centre National de Transfusion Sanguine, Paris, France), soit du glucagon- ${ }^{125}$ I avec des radioactivités spécifiques de l'ordre de $260 \mu \mathrm{Ci} / \mu \mathrm{g}$ (Sorin, Turin, Italie).

\section{Antisérum anti-glucagon}

L'antisérum a été obtenu par immunisation d'un lapin. L’immunisation initiale a été induite par 3 injections sous-cutanées, effectuées à 3 semaines d'intervalle, de $3 \mathrm{mg}$ de glucagon couplés à de l'albumine d'oeuf et émulsionnés dans de l'adjuvant de Freund selon la technique de Unger et al. [20]. L'immunisation ultérieure a été poursuivie en administrant tous les 2 mois des doses de rappel par voie intramusculaire. Chacune de ces doses de rappel était composée de 5 injections, effectuées à 2 jours d'intervalle, de $1 \mathrm{mg}$ de glucagon émulsionné dans un mélange d'adjuvant de Freund et de cire d'abeille liquéfiée selon la technique de Worobee et al. [24].

L'antisérum a été prélevé par ponction cardiaque 3 semaines après la 2ème injection de rappel.

\section{Incubation}

Les réactifs employés sont les suivants:

- tampon véronal $0.05 \mathrm{M}, \mathrm{pH} 8.6$, additionné de $0.25 \%$ d'albumine bovine (fraction V, Sigma Chemical Company, St. Louis, Missouri). Une solution frâche de tampon albuminé est préparée pour chaque incubation à partir d'une solution stock de tampon véronal;

- Trasylol (inhibiteur des protéinases) à 100000 $\mathrm{KIU} / 10 \mathrm{ml}$ ou $500000 \mathrm{KIU}$ sous forme lyophylisée;

- solutions de glucagon- ${ }^{131} \mathrm{I}$ ou de glucagon-125I préparées dans du tampon véronal albuminé et contenant 0.060 à $0.070 \mathrm{ng}$ de traceur $/ 50 \mu 1$;

- antisérum dilué à $1: 550$ avec du tampon véronal albuminé;

-2 solutions standard de glucagon à 1 et $10 \mathrm{ng} / \mathrm{ml}$ et préparées dans du tampon véronal albuminé contenant $1000 \mathrm{KIU}$ de Trasylol/ml,
Les solutions stock de tampon véronal et le Trasylol sont conservés à $4^{\circ} \mathrm{C}$. Les solutions de glucagon marqué et non marqué, l'antisérum dilué et les échantillons sont conservés à $-20^{\circ} \mathrm{C}$.

Pendant toute la durée de la préparation du dosage les tubes d'incubation sont maintenus dans un bain glacé. Les réactifs y sont ajoutés dans l'ordre suivant:

a) tampon véronal albuminé,

b) Trasylol $100 \mu \mathrm{l}$ (1000 KIU),

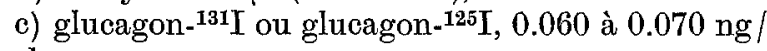
$50 \mu \mathrm{l}$,

d) antisérum $1: 550,50 \mu 1$,

e) standards de glucagon, 0.020 à $1 \mathrm{ng}$, et échantillons, $100 \mu 1$.

Le volume d'incubation final est de $600 \mu$ l. Des tubes contrôles, sans antisérum, correspondant à la courbe standard et aux divers échantillons sont également inclus dans l'expérience. Les tubes sont recouverts de parafilm, agités sur Vortex et incubés à $4^{\circ} \mathrm{C}$. Les réactions antigène - anticorps atteignent leur équilibre en 3 jours, en agitation constante. Tous les dosages sont effectués en double.

4. Séparation du glucagon marqué libre et du glucagon marqué liè à l'anticorps par le charbon-dextran

La séparation est réalisée par l'adsorption du glucagon marqué libre sur le charbon-dextran. La suspension de charbon-dextran est préparée selon les indications de Herbert et al. [9] mais en réduisant de moitié la concentration en charbon de la suspension $(12.5 \mathrm{~g} / 1)$. Dans chaque tube sont ajoutés 50 à $150 \mu l$ de sérum de boeuf, de manière à amener la concentration en protéines plasmatiques à une même valeur dans chaque tube, puis $2 \mathrm{ml}$ de la suspension de charbon-dextran. Les tubes sont ensuite individuellement agités sur Vortex pendant $10 \mathrm{sec}$ puis centrifugés à 2600 tours/ minute pendant $25 \mathrm{~min}$. Le surnageant est décanté immédiatement après la centrifugation et seuls les tubes contenant le sédiment de charbon sont comptés. Toutes les opérations (adjonction de sérum et de charbondextran, agitation, centrifugation et décantation) sont effectuées à $4^{\circ} \mathrm{C}$.

Les comptages sont réalisés à l'aide d'un scintillateur gamma automatique à cristal (Philips).

\section{Expression des résultats}

Les résultats sont exprimés dans le texte, les tableaux et les figures par la valeur moyenne, la déviation standard: $s=\sqrt{(x-\bar{x})^{2} /(n-1)}$, et le coefficient de variation: $\mathrm{CV}=s /$ moyenne $(\%)$.

\section{Résultats et estimation de la méthode de dosage}

\section{Capacité d'adsorption du charbon-dextran}

Dans les tubes contrôles, en absence d'antisérum, le pourcentage de la radioactivité totale adsorbée sur le charbon-dextran varie entre 80 et $96 \%$ suivant les lots de glucagon marqué et le temps écoulé depuis le 
marquage. (Nous admettrons, dans la suite de cet article, que ce pourcentage de radioactivité adsorbée en l'absence d'antisérum représente la quantité de traceur non endommagé). La concentration de charbondextran utilisée est suffisante pour adsorber le traceur non endommagé car l'adjonction de glucagon non marqué, en doses allant jusqu'à $1000 \mathrm{ng}$, n'interfère pas significativement avec cette adsorption (fig. 1).

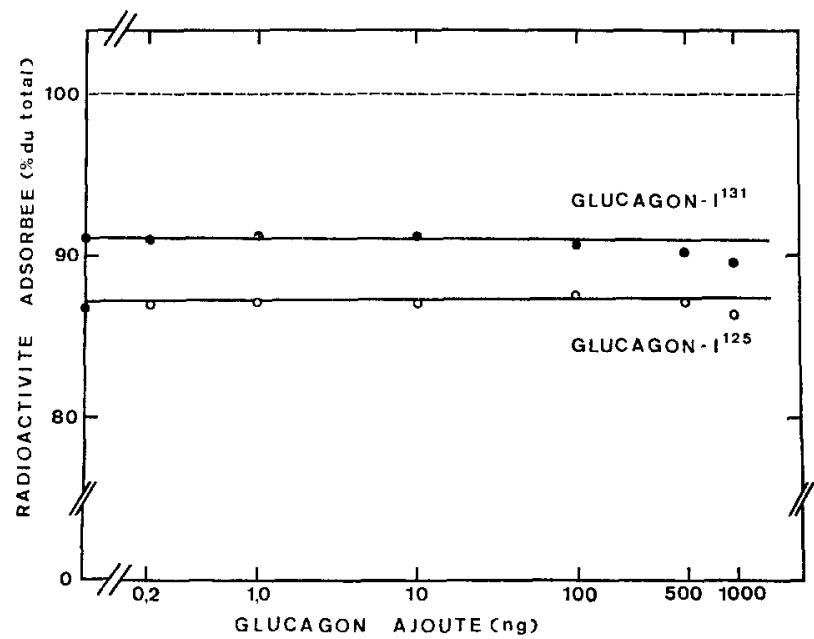

Fig. 1. Adsorption sur le charbon-dextran de $0.060 \mathrm{ng}$ de glucagon marqué, en l'absence d'antisérum et en présence de quantités croissantes de glucagon non marqué.

\section{Capacité de liaison de l'antisérum}

Nous définissons la capacité de liaison de l'antisérum par le pourcentage de traceur non endommagé que $50 \mu 1$ d'antisérum non dilué ou dilué sont capables de lier dans notre système de dosage. La formule utilisée pour le calcul de cette capacité de liaison est la suivante:

liaison glucagon marqué $(\%)=\frac{F-F^{\prime}}{F^{\prime}}$

où $F=$ radioactivité du charbon-dextran dans les tubes contrôles, en absence d'antisérum,

et $F^{\prime}=$ radioactivité du charbon-dextran dans les tubes correspondants, en présence d'antisérum.

Pour un lot de glucagon donné, la capacité de liaison de l'antisérum (ou pourcentage de traceur non endom. magé immunoréactif) diminue en général légèrement avec le temps écoulé depuis le marquage de l'hormone. Cette diminution est néanmoins trop faible pour affecter la reproductibilité de la courbe standard pendant une période déterminée. La courbe standard moyenne obtenue pour 11 essais réalisés au cours de 7 semaines consécutives (fig. 2), et dont l'analyse de précision est reportée dans le tableau 1, illustre ce fait.

Par contre, la capacité de liaison de l'antisérum peut varier considérablement avec les différents lots de glucagon marqué (fig. 3). Le pourcentage de liaison maximum que nous avons observé est de 90\% (fig. 3, lot A de glucagon-131I) et l'incubation avec des quantités croissantes (allant jusqu'à $200 \mu \mathrm{l}$ ) d'antisérum non dilué n'a pu augmenter la valeur de cette liaison. Il semble donc bien que, dans nos conditions expéri-

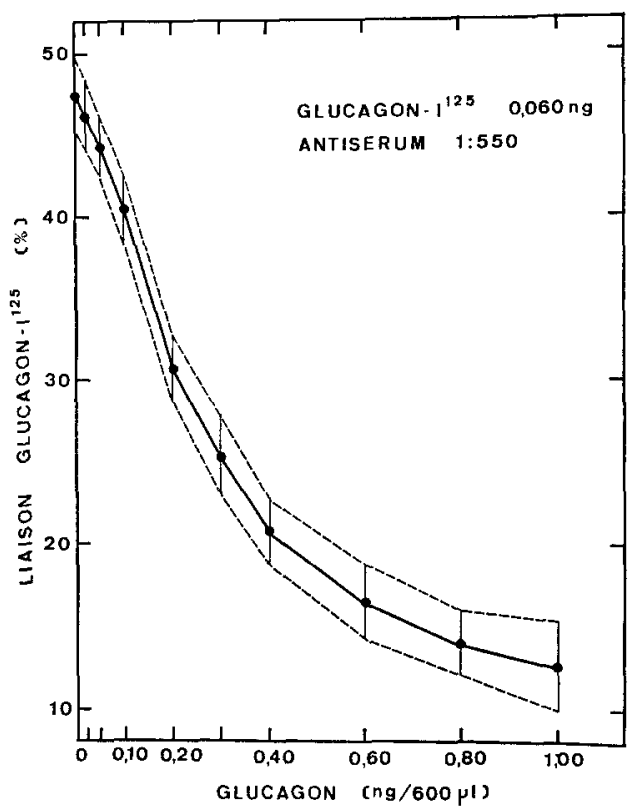

Fig. 2. Courbe standard moyenne obtenue pour 11 essais réalisés au cours de 7 semaines consécutives. Les courbes en traits discontinus représentent les limites de la zone fiduciaire $95 \%(\bar{x} \pm \mathrm{t} / s)$.

Tableau 1. Analyse de la précision des mesures de la courbe standard de la fig. 2.

\begin{tabular}{|c|c|c|c|}
\hline $\begin{array}{l}\text { Glucagon } \\
\text { non marqué } \\
\text { ajouté } \\
(\mathrm{ng} / 600 \mu \mathrm{l})\end{array}$ & $\begin{array}{l}\text { Liaison } \\
\text { glucagon-125I } \\
\text { (\% du traceur } \\
\text { non endommagé) }\end{array}$ & $\begin{array}{l}\text { Ecart-type } \\
(s)\end{array}$ & $\begin{array}{l}\text { Coefficient } \\
\text { de } \\
\text { variation } \\
\text { (CV) }\end{array}$ \\
\hline 0 & 47.34 & 1.15 & 2.4 \\
\hline 0.02 & 46.35 & 1.01 & 2.2 \\
\hline 0.05 & 44.38 & 0.85 & 1.9 \\
\hline 0.10 & 40.61 & 0.88 & 2.2 \\
\hline 0.20 & 30.80 & 0.99 & 3.2 \\
\hline 0.30 & 25.40 & 1.02 & 4.0 \\
\hline 0.40 & 20.81 & 0.89 & 4.3 \\
\hline 0.60 & 16.53 & 0.97 & 5.9 \\
\hline 0.80 & 13.98 & 0.85 & 6.1 \\
\hline 1.00 & 12.65 & 1.24 & 9.8 \\
\hline
\end{tabular}

Les valeurs des pourcentages de liaison représentent la moyenne des points obtenus en double au cours de 11 essais consécutifs.

mentales, la capacité de liaison de l'antisérum dépende principalement des caractéristiques de l'hormone marquée et non des qualités de l'antisérum lui-même.

Il est évident que la variation de la capacité de liaison de l'antisérum va entraîner une variation de l'aspect des courbes standard (fig. 4). Malgré ce fait, il est remarquable de constater que les concentrations en 
glucagon de divers échantillons (sérums de plusieurs espèces, milieux d'incubation d'îlots isolés de rat) restent très comparables (tableau 2 ).

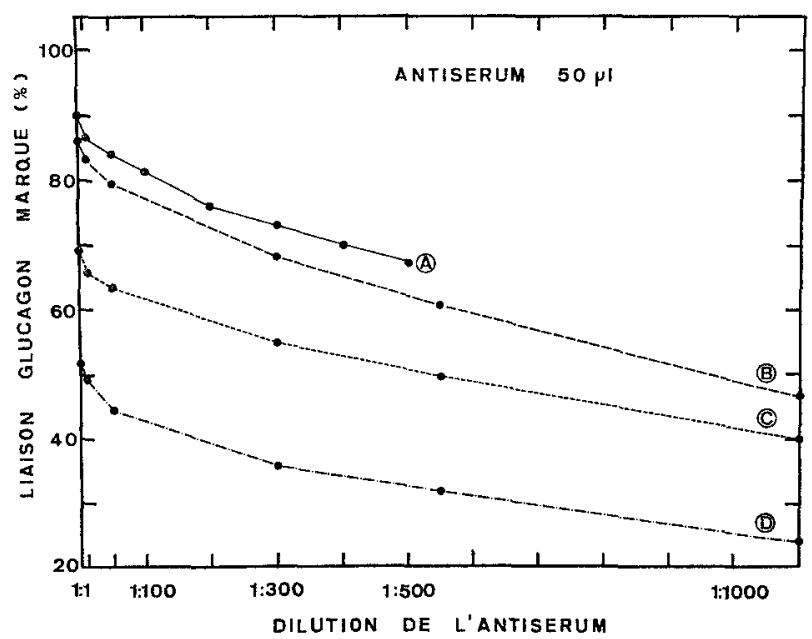

Fig. 3. Variation de la capacité de liaison de l'antisérum pour différents lots de glucagon marqué; $(\mathrm{A})$ et $(\mathrm{B})=$ lots de glucagon-131I, (C) et $(\mathrm{D})=$ lots de glucagon- ${ }^{125} \mathrm{I}$.

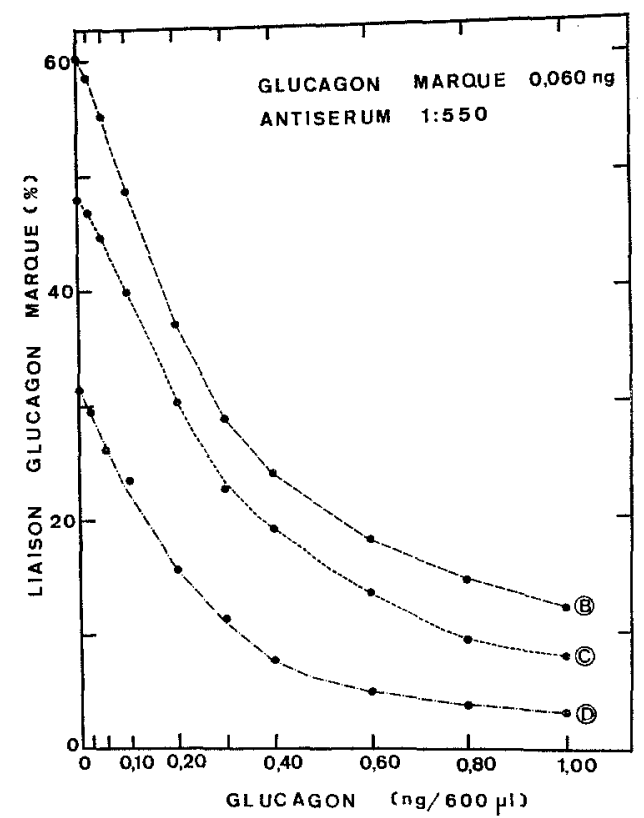

Fig. 4. Courbes standard obtenues avec différents lots de glucagon marqué; $(A)=$ lot de glucagon ${ }^{131} \mathbf{I},(\mathrm{C})$ et (D) $=$ lots de glucagon ${ }^{125}$ I (cf. fig. 3 ).

\section{Efficacité du Trasylol}

Nous avons vérifié que l'emploi de Trasylol empêche la dégradation du traceur incubé en présence de sérum ou de plasma en ajoutant aux tubes d'incubation un excès d'antisérum. Le tableau 3 montre les résultats obtenus avec un lot de glucagon-131 I. L'excès d'antisérum lie autant de traceur non endommagé en l'absence qu'en présence de sérum ou de plasma. Il n'y a donc pas, dans nos conditions expérimentales, de diminution de la fraction immunoréactive du traceur au cours de l'incubation. Cette absence de dégradation exclut l'éventualité d'obtenir des glucagonémies faussement élevées, ce qui est le cas lorsqu'une dégradation du traceur est interprétée comme un déplacement du traceur par de l'hormone non marquée [5].

Tableau 2. Concentrations en glucagon ( $\mathrm{g} / \mathrm{ml}$ ) de divers échantillons dosés avec différents lots de glucagon marqué (cf. fig. 3 et fig. 4 )

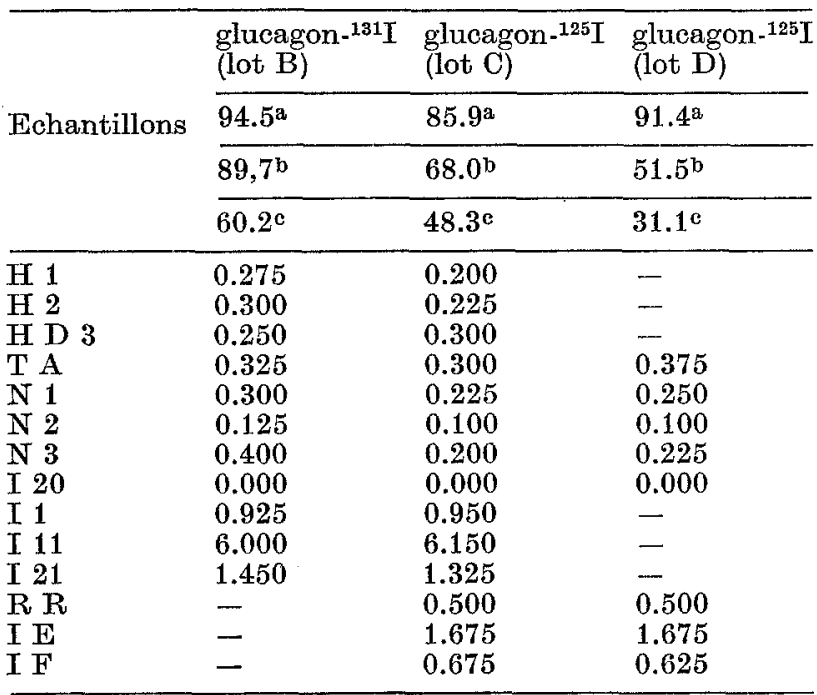

a Adsorption de radioactivité sur le charbon-dextran dans les tubes contrôles, en absence d'antisérum = traceur non endommagé ( $\%$ du total).

b Liaison du glucagon marqué en présence d'un excès d'antisérum (\% du traceur non endomagé).

c Liaison du glucagon marqué par de l'antisérum 1:550 (\% du traceur non endommagé).

Tableau 3.

\begin{tabular}{lll}
\hline Echantillons & $\begin{array}{l}\text { Traceur non } \\
\text { endommagé } \\
\text { (\% du total) }\end{array}$ & $\begin{array}{l}\text { Traceur } \\
\text { immunoréactif } \\
\text { (\% du traceur } \\
\text { non endommagé) }\end{array}$ \\
\hline $\left.\begin{array}{l}\text { Traceur seul } \\
\begin{array}{l}\text { Traceur + plasma } \\
\text { humain }(100 \mu l)\end{array} \\
\begin{array}{l}\text { Traceur + sérum de } \\
\text { rat }(100 \mu l)\end{array}\end{array}\right)$ 93.1 & 89.7 \\
$\begin{array}{c}\text { Traceur + plasma de } \\
\text { canard }(100 \mu l)\end{array}$ & 89.5 & 90.0 \\
\hline
\end{tabular}

Une légère dégradation peut cependant se produire, en particulier avec du sérum de rat qui possède une activité protéolytique très élevée [12], lorsque l'incubation est effectuée à température ambiante. C'est la raison pour laquelle nous maintenons les tubes dans un bain glacé pendant la préparation du dosage.

En ce qui concerne l'effet du. Trasylol ajouté au sang au moment du prélèvement, nous avons constaté, comme d'autres auteurs $[5,7]$, que la concentration en 
glucagon endogène d'échantillons prélevés sur Trasylol n'était pas significativement différente de celle d'échantillons prélevés sans Trasylol. De plus, la décongélation ne semble pas affecter le taux de glucagon endogène mesuré. En effet, la concentration en glucagon d'un plasma humain dosé 5 fois (frais et après 4 décongélations successives) est de $0.270 \mathrm{ng} / \mathrm{ml}$ avec Trasylol $(s=0.033 ; n=5$ ) et de $0.230 \mathrm{ng} / \mathrm{ml}$ sans Trasylol $(s=0.033 ; n=5)$, sans variation systématique au cours du temps. Dans les mêmes conditions nous arons obtenu $0.245 \mathrm{ng} / \mathrm{ml}(s=0.045 ; n=5)$ et 0.240 $\mathrm{ng} / \mathrm{ml}(s=0.045 ; n=5)$ pour un plasma de chien.

\section{Quelques problèmes techniques liès à la séparation par le charbon-dextran}

Nous avons constaté que le charbon-dextran, aux concentrations protéiques basses, adsorbe non seulement le glucagon marqué libre mais aussi une partie du glucagon lié à l'anticorps. L'adjonetion de sérum de boeuf (ou de tout autre sérum ou plasma) immédiatement avant la suspension de charbon-dextran prévient cette adsorption. Il est donc essentiel de réaliser une même concentration finale en protéines plasmatiques dans tous les tubes. En effet, une teneur trop faible en protéines plasmatiques dans les tubes de la courbe standard amènerait une estimation, erronnée et trop faible, de la concentration en glucagon des échantillons plasmatiques (fig. 5).

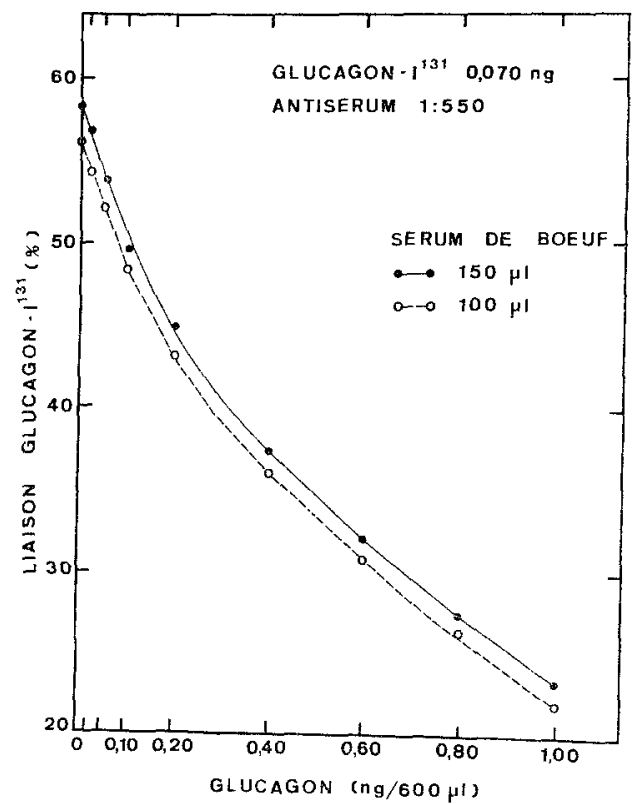

Fig. 5. Influence de la concentration en protéines plasmatiques sur l'adsorption de radioactivité par le charbondextran.

De même, la centrifugation à température ambiante augmente fortement l'adsorption de radioactivité par le charbon-dextran (fig. 6). Cette augmentation d'adsorption se produit également pour les tubes contenant les échantillons et l'estimation de leur concentration en glucagon n'en est, par conséquent, pas faussée lorsque ces tubes sont centrifugés en même temps que la courbe standard correspondante. Cependant, la perte de sensibilité de la courbe standard, le manque de reproductibilité des résultats obtenus dans ces conditions et l'impossibilité de comparer les résultats obtenus au cours de centrifugations successives nous ont conduit à effectuer toutes les manipulations de la séparation par le charbon-dextran à $4^{\circ} \mathrm{C}$.

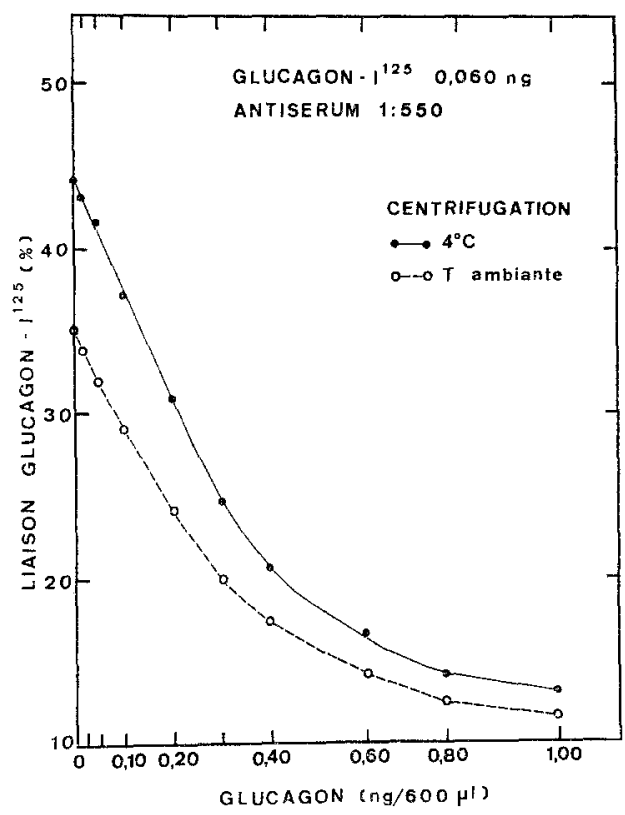

Fig. 6. Influence de la température de centrifugation sur l'adsorption de radioactivité par le charbon-dextran.

\section{Evaluation de la méthode}

a) Sensibilité. La sensibilité a été étudiée à l'aide des points obtenus au cours de 11 courbes standard successives (fig. 3 et tableau 3). Le point $0.02 \mathrm{ng}$ est très significativement différent de zéro (différences pairées: $t=7.71, n=11, P<0.001$ ) et correspond à une concentration en glucagon plasmatique de 0.200 $\mathrm{ng} / \mathrm{ml}$. En fait, comme nous le verrons dans le paragraphe suivant la sensibilité de la méthode est égale à environ $0.100 \mathrm{ng} / \mathrm{ml}$ de plasma.

b) Précision. La précision de la méthode a été évaluée par deux procédés:

- d'une part, en estimant l'écart-type des pourcentages de liaison et des concentrations en glucagon correspondantes d'échantillons ( 4 concentrations différentes) dosés 10 fois au cours d'un même essai (tableau 4);

- d'autre part, en estimant l'écart-type pour 50 paires de dosages (6 gammes différentes) effectuées au cours d'essais successifs; dans ce cas, l'écart-type a été calculé selon la formule $\mathrm{s}=\sqrt{\frac{d^{2}}{2 n}}[19]$, dans laquelle 
d représente la différence entre les 2 valeurs de chaque paire et $n$ le nombre de paires (tableau 5 ).

Ainsi que le montre le tableau 4, l'erreur attachée au pourcentage de liaison à l'intérieur d'un essai est très faible. Elle est due en majeure partie à l'erreur inhérente au comptage, cette dernière étant estimée par 10 comptages successifs de chaque tube (tableau 4 , nombre entre parenthèses) et ne dépassant générale. ment pas 1 pour cent du nombre de coups donnés par le compteur. Traduits en termes de concentration glucagonique ( $\mathrm{ng} / \mathrm{ml}$ ), les écarts-types sont comparables aux concentrations comprises entre 0 et $2.000 \mathrm{ng} / \mathrm{ml}$, puis augmentent pour les valeurs supérieures à 2.000 $\mathrm{ng} / \mathrm{ml}$ (tableau 5). Il est remarquable que, pour une concentration donnée, la précision des estimations est la même à l'intérieur d'un essai (tableau 4) et au cours d'essais successifs (tableau 5). de la formule $t=d \sqrt{\left(s_{1}^{2} / n_{1}\right)+\left(s_{2}^{2} / n_{2}\right)}$ indique que 2 valeurs ne seront significativement différentes au seuil $\mathrm{P}=0.05$ que si la différence entre les 2 valeurs excède $0.200 \mathrm{ng} / \mathrm{ml}$ dans la gamme de concentration 0 à 2.000 $\mathrm{ng} / \mathrm{ml}$ et $0.350 \mathrm{ng} / \mathrm{ml}$ pour les valeurs supérieures. De même, la plus petite valeur significativement différente de zéro est d'environ $0.100 \mathrm{ng} / \mathrm{ml}$.

c) Reproductibilité. Différents échantillons ont été dosés à $n$ reprises dans des essais successifs, pendant une période de 4 mois. Les valeurs de concentration en glucagon obtenues sont de $0.211 \mathrm{ng} / \mathrm{ml}$ pour un plasma humain $(s=0.048, n=17)$, de $0.334 \mathrm{ng} / \mathrm{ml}$ pour un sérum de rat ( $s=0.062, n=14$ ), de $0.513 \mathrm{ng} / \mathrm{ml}$ pour un plasma de rat ( $s=0.079, n=8$ ), et de $1.635 \mathrm{ng} / \mathrm{ml}$ pour un milieu d'incubation d'îlots de pancréas de rat $(s=0.072, n=12)$.

Tableau 4. Précision des mesures à l'intérieur d'un dosage.

\begin{tabular}{|c|c|c|c|c|c|c|c|}
\hline \multirow[t]{2}{*}{ Echantillons } & \multicolumn{3}{|c|}{$\begin{array}{l}\text { Liaison glucagon- }{ }^{-125} \mathrm{I} \\
\text { (\% du traceur non } \\
\text { endommagé) }\end{array}$} & \multicolumn{3}{|c|}{$\begin{array}{l}\text { Concentration en glucagon } \\
(\mathrm{ng} / \mathrm{ml})\end{array}$} & $\begin{array}{l}\text { Erreur due aux } \\
\text { comptages }(\%)\end{array}$ \\
\hline & $\begin{array}{l}\text { Mesure } \\
\text { moyenne }\end{array}$ & $s$ & $\mathrm{CV}$ & $\begin{array}{l}\text { Mesure } \\
\text { moyenne }\end{array}$ & $s$ & $\mathrm{CV}$ & $\frac{(\mathrm{CV})}{\mathrm{CV}}=\frac{(s)}{s}$ \\
\hline $\begin{array}{l}1 \\
\text { plasma humain }\end{array}$ & $(\stackrel{38.72}{(")}$ & $\begin{array}{c}0.50 \\
(0.37)\end{array}$ & $\begin{array}{c}1.29 \\
(0.95)\end{array}$ & $(\stackrel{0.166}{)}$ & $\begin{array}{c}0.073 \\
(0.054)\end{array}$ & $\begin{array}{c}43.97 \\
(32.53)\end{array}$ & 74.0 \\
\hline $\begin{array}{l}2 \\
\text { sérum de rat }\end{array}$ & $(\not 2,11$ & $\begin{array}{c}0.63 \\
(0.48)\end{array}$ & $\begin{array}{c}1.70 \\
(1.28)\end{array}$ & $(\because, 407$ & $\begin{array}{c}0.097 \\
(0.073)\end{array}$ & $\begin{array}{c}23.83 \\
(17.93)\end{array}$ & 75.3 \\
\hline $\begin{array}{l}3 \\
\text { incubation } \\
\text { illots de rat }\end{array}$ & $(" 35.35$ & $\begin{array}{c}0.60 \\
(0.44)\end{array}$ & $\begin{array}{c}1.69 \\
(1.24)\end{array}$ & $\begin{array}{l}0.684 \\
(")\end{array}$ & $\begin{array}{c}0.090 \\
(0.066)\end{array}$ & $\begin{array}{c}13.16 \\
(9.65)\end{array}$ & 73.3 \\
\hline $\begin{array}{l}4 \\
\text { incubation } \\
\text { îlots de rat }\end{array}$ & $(" 31,40$ & $\begin{array}{c}0.68 \\
(0.43)\end{array}$ & $\begin{array}{c}2.16 \\
(1.37)\end{array}$ & $(\stackrel{1.274}{)}$ & $\begin{array}{c}0.085 \\
(0.054)\end{array}$ & $\begin{array}{c}6.67 \\
(4.24)\end{array}$ & 63.5 \\
\hline
\end{tabular}

Tableau 5. Précision des mesures pour l'ensemble du dosage. Résultats obtenus par l'analyse de 50 paires de dosages dans 6 gammes de mesures.

\begin{tabular}{llll}
\hline $\begin{array}{l}\text { Gammes de } \\
\text { concentration } \\
\text { en glucagon } \\
(\mathrm{ng} / \mathrm{ml})\end{array}$ & $\begin{array}{l}\text { Mesure } \\
\text { moyenne } \\
(\mathrm{ng} / \mathrm{ml})\end{array}$ & $\begin{array}{l}\text { Ecart-type } \\
(s)\end{array}$ & $\begin{array}{l}\text { Coefficient } \\
\text { de } \\
\text { variation } \\
(\mathrm{CV})\end{array}$ \\
\hline $0 \quad-0.250$ & $\mathbf{0 . 1 6 6}$ & 0.064 & $\mathbf{3 5 . 0}$ \\
$0.250-0.500$ & 0.387 & 0.072 & 18.5 \\
$0.500-1.000$ & 0.688 & 0.073 & 10.5 \\
$1.000-1.500$ & $\mathbf{1 . 2 0 7}$ & 0.092 & 7.6 \\
$1.500-2.000$ & $\mathbf{1 . 7 1 5}$ & 0.088 & 5.1 \\
$2.000-6.000$ & $\mathbf{3 . 3 7 2}$ & 0.137 & 4.0 \\
\hline
\end{tabular}

Ces erreurs sont inévitables dans tous les dosages immunologiques puisqu'elles dépendent essentiellement de la précision du comptage lui-même. Il n'en reste pas moins que ces observations incitent à la prudence lorsqu'on en vient à dire que 2 glucagonémies mesurées au cours d'un même essai sont statistiquement différentes. Ainsi, dans nos conditions expérimentales où les analyses sont faites en double, l'emploi
Les erreurs liées à ces déterminations n'excèdent pas celles de la méthode et nous n'avons pas observé de variation systématique au cours du temps.

d) Récupérations. Des doses croissantes de glucagon non marqué (de 0 à 5.000 ng en solution dans $50 \mu \mathrm{l}$ de tampon véronal albuminé à $1000 \mathrm{KIU}$ de Trasylol $/ \mathrm{ml}$ ) et $1000 \mathrm{KIU}$ de Trasylol (dans un volume de $20 \mu \mathrm{l}$ ) ont été ajoutés à $930 \mu 1$ de plasma humain. La concentration en glucagon de ces préparations a été estimée sur $100 \mu \mathrm{l}$ et exprimée en $\mathrm{ng} / \mathrm{ml}$. Les récupérations obtenues au cours de 7 essais consécutifs sont d'environ $90 \%$ (tableau 6).

La récupération satisfaisante du glucagon a été confirmée par des expériences où nous avons injecté, par voie intraveineuse, du glucagon aux doses de 1.25 et $10 \mu \mathrm{g} / \mathrm{kg}$ à des canards (fig. 7). La concentration en glucagon du plasma au temps 1 min est approximativement 10 fois plus élevée dans le cas où la forte dose a été injectée. A propos de ces expériences, notons que le niveau de la glucagonémie ainsi induite conditionne 
d'ailleurs l'ampleur de la riposte hyperglycémique et hyperinsulinémique [17]. Le temps de demi-disparition du glucagon injecté est très rapide, de l'ordre de 2 à 3

Tableau 6. Récupération du glucagon non marqué ajouté à du plasma humain. Moyennes de 7 expériences consécutives.

\begin{tabular}{llcc}
\hline $\begin{array}{l}\text { Glucagon } \\
\text { ajouté } \\
(\mathrm{ng} / \mathrm{ml})\end{array}$ & $\begin{array}{l}\text { Concentration } \\
\text { en glucagon }\end{array}$ & \multicolumn{2}{l}{ Glucagon récupéré } \\
$(\mathrm{ng} / \mathrm{ml} \pm s)$ & $(\mathrm{ng} / \mathrm{ml} \pm s)$ & $(\% \pm s)$ \\
\hline 0 & $0.186 \pm 0.050$ & - & - \\
0.250 & $0.425 \pm 0.012$ & $0.239 \pm 0.020$ & $95.6 \pm 7.9$ \\
0.500 & $0.639 \pm 0.056$ & $0.453 \pm 0.037$ & $90.6 \pm 7.3$ \\
1.000 & $1.092 \pm 0.061$ & $0.907 \pm 0.099$ & $90.7 \pm 9.9$ \\
2.000 & $2.028 \pm 0.142$ & $1.842 \pm 0.122$ & $92.1 \pm 6.1$ \\
5.000 & $4.801 \pm 0.330$ & $4.615 \pm 0.339$ & $92.3 \pm 6.8$ \\
\hline
\end{tabular}

min, jusqu'au moment où les concentrations en glucagon retournent aux valeurs normales (1 à 15 min dans le cas de la dose élevée et 1 à 5 min dans le cas de la dose faible).

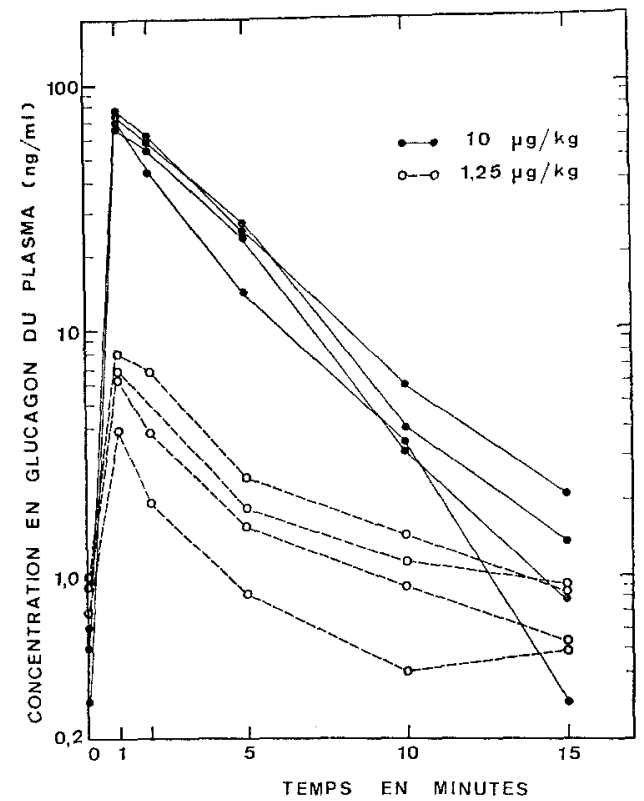

Fig. 7. Concentrations en glucagon du plasma après injection intraveineuse de glucagon de boeuf-porc aux doses de 1.25 et $10 \mu \mathrm{g} / \mathrm{kg}$ chez le canard.

e) Spécificité. Des extraits acido-alcooliques de pancréas de chien, de rat, de cobaye et de canard contiennent une substance qui possède les mêmes propriétés immunologiques que le glucagon de boeufporc purifié (fig. 8). Ces observations confirment celles qui ont été faites par d'autres auteurs $[16,20,18,3]$ montrant que les glucagons pancréatiques de différentes espèces ont un comportement immunologique comparable.

L'antisérum que nous utilisons donne également des courbes de dilution qui sont parallèles à celles du glucagon de boeuf-porc pour des extraits acido-alcooliques du duodénum chez ces mêmes espèces (fig. 8). La concentration de la substance présente dans les extraits duodénaux ne représente cependant, par poids de tissu frais, que 0.2 à $3 \%$ de la quantité de glucagon extraite du pancréas correspondant. Contrairement aux observations qui ont été faites par d'autres auteurs $[16,20,18,4]$, le comportement immunologique de cette substance est comparable dans notre système à celui du glucagon pancréatique. Ces données contradictoires confirment que les anticorps anti-glucagon (obtenus selon les lois du hasard) présentent des différences, peut-être plus quantitatives que qualitatives, en ce qui concerne leurs propriétés de discrimination entre le glucagon pancréatique et les substances "glucagon-like« d'origine intestinale $(8, \mathrm{I})$.

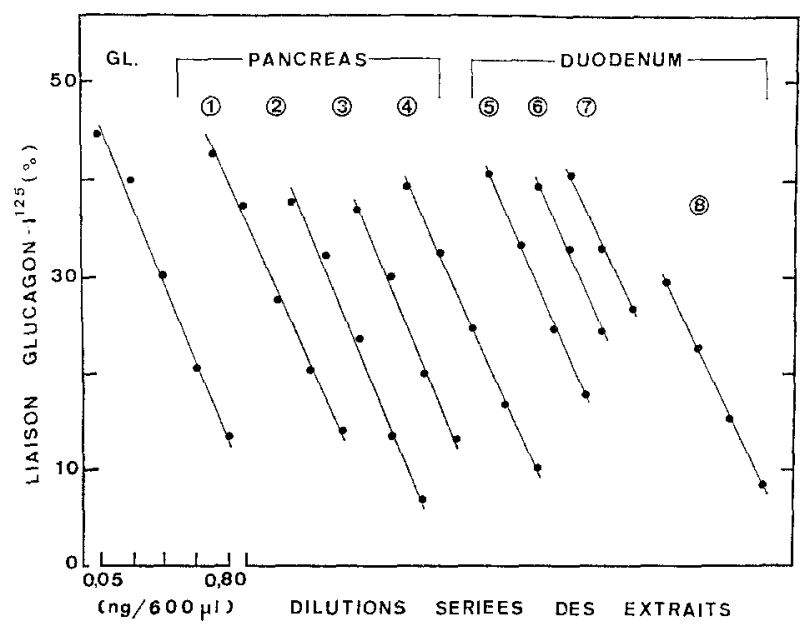

Fig. 8. Courbes d'inhibition de la liaison antigène-anticorps par du glucagon purifié de boeuf-porc (GL.), des extraits acido-alcooliques de pancréas de chien (1), de rat (2), de cobaye (3), de canard (4), et des extraits acido. alcooliques de duodénum de chien (5), de rat (6), de cobaye (7), et de canard (8). Les doses de glucagon purifié et les dilutions sont portées sur une échelle logarithmique.

Nous avons également étudié la spécificité du dosage en effectuant des dilutions sériées d'un milieu d'incubation d'îlots de pancréas de rat et de divers échantillons de sérum ou de plasma. Le tableau 7 montre que la concentration apparente en glucagon, exprimée en $\mathrm{ng} / \mathrm{ml}$ d'échantillon non dilué, est identique pour les diverses dilutions, qu'il s'agisse du milieu d'incubation, de plasma de chien ou de sérum de rat. Il ne semble done pas y avoir d'interférence autre que celle de la substance d'origine duodénale dans l'estimation de la concentration en glucagon plasmatique dans nos conditions expérimentales. Par contre, l'augmentation apparente de la concentration en glucagon du plasma de canard indique une autre interférence, peut-être celle des gamma-globulines comme l'ont suggéré Unger et Eisentraut [21] et Assan et al. [4].

\section{Valeurs expérimentales}

Nous avons estimé la concentration en glucagon dans le plasma ou le sérum veineux périphérique de 
différentes espèces. Chez des sujets normaux à jeun, elle est de $0.208 \mathrm{ng} / \mathrm{ml}(s=0.120, n=29)$; chez le chien anesthésié à jeun, elle est de $0.396 \mathrm{ng} / \mathrm{ml}(s=$ $0.270, n=15$ ); chez le rat nourri, dans le sérum obtenu par décapitation, elle est de $0.354 \mathrm{ng} / \mathrm{ml}(s=0.144$, $n=5$ ) et chez le canard à jeun, elle est de $0.909 \mathrm{ng} / \mathrm{ml}$ $(s=0.305, n=30)$.
2. Assan, R., Rosselin, G., Tchobroutsky, G., Drouet, J., Dolais, J., Dérot, M.: Dosage radioimmunologique du glucagon plasmatique chez l'homme. Diabetologia 2, 136 (1966).

3. - Glucagon and glucagon cross-reacting substances in gastric (GE) and pancreatic extracts (PE) for 12 animal species. 3rd International Congress of Endocrinology, Excerpta Medica Foundation, Inter-

Tableau 7. Effet de la dilution sur la concentration apparente en glucagon de divers échantillons.

\begin{tabular}{llllll}
\hline \multirow{2}{*}{$\begin{array}{l}\text { Dilution } \\
\text { de } \\
\text { l'échantillon }\end{array}$} & \multicolumn{3}{l}{ Concentration en glucagon } & \multicolumn{2}{l}{ (ng/ml d'échantillon non dilué) } \\
\cline { 2 - 6 } & $\begin{array}{l}\text { incubation } \\
\text { ilots } \\
\text { de rat }\end{array}$ & $\begin{array}{l}\text { plasma } \\
\text { humain }\end{array}$ & $\begin{array}{l}\text { plasma } \\
\text { de } \\
\text { chien }\end{array}$ & $\begin{array}{l}\text { sérum } \\
\text { de } \\
\text { rat }\end{array}$ & $\begin{array}{l}\text { plasma } \\
\text { de } \\
\text { canard }\end{array}$ \\
\hline $1: 1$ & $\mathbf{1 . 1 5 0}$ & 0.220 & 0.342 & 0.350 & 0.810 \\
$1: 2$ & 1.100 & 0.266 & 0.300 & 0.400 & 0.992 \\
$1: 4$ & 1.000 & 0.264 & 0.364 & 0.332 & 1.680 \\
$1: 8$ & 1.000 & - & - & - & 1.680 \\
\hline
\end{tabular}

\section{Conclusion}

La méthode de dosage radioimmunologique que nous présentons semble très valable pour la mesure de faibles concentrations en glucagon. Elle a l'avantage de comprendre une séparation simple et rapide de l'antigène marqué libre et de l'antigène marqué lié à l'anticorps. Son emploi, à température basse $\left(4^{\circ} \mathrm{C}\right)$ et en présence d'une concentration en protéines plasmatiques suffisante, ne présente pas de difficultés majeures. En raison du type d'antisérum que nous possédons et qui réagit immunologiquement avec une substance d'origine duodénale, notre méthode semble actuellement limitée à l'étude de la sécrétion du glucagon dans des expériences effectuées in vitro. L'importance de l'interférence de cette substance dans l'estimation des concentrations en glucagon du plasma reste cependant à déterminer.

Remerciements: Le présent travail a bénéficié d'une aide financière accordée à l'un d'entre nous (V.L.-M.) par le Service du Fonds de la Recherche, Délégation Générale à la Recherche Scientifique et Technique, Paris (France), d'un Crédit aux Chercheurs du Fonds National de la Recherche Scientifique de Belgique (W.J.M.), et du contrat Euratom-Universités de Pise et de Bruxelles $(026-63-4-$ BIAC). Nous tenons à témoigner notre gratitude à Monsieur le Professeur F. Stutinsky, Directeur du Laboratoire de Physiologie de l'Université de Strasbourg, pour l'intérêt et les encouragements qu'il nous a témoignés au cours de ce travail. Nous tenons également à remercier très vivement le Dr. Mary Root (Lilly Research Laboratories, Indianapolis, USA) pour le don généreux de glucagon purifié, Monsieur Goffinet (Bayer, Belgique) pour le don du Trasylol et le Dr. U. Rosa (Sorin, Saluggia) pour les préparations de glucagon-125I.

\section{Bibliographie}

1. Aguilar-Parada, E., Eisentraut, A. M., Unger, R. H.: Pancreatic glucagon secretion in normal and diabetic subjects. Amer. J. med. Sci. 257, 415-418 (1969). national Congress Series n ${ }^{\circ} 157$ (Abstract $n^{\circ}$ 107, p. 43), Mexico (1968).

4. - Rosselin, G., Tchobroutsky, G., Freychet, P.: Dosage radioimmunologique du glucagon; application à l'étude d'extraits digestifs humains et animaux. Path. Biol. 17, 45-51 (1969).

5. Eisentraut, A.M., Whissen, N., Unger, R.H.: Incubation damage in the radioimmnoassay for human plasma glucagon and its prevention with "Trasylol". Amer. J. med. Sci. 255, 137-142 (1968).

6. Grodsky, G.M., Hayashida, T., Peng, C.T., Geschwind, I.I.: Production of glucagon antibodies and their role in metabolism and immunoassay of gluca. gon. Proc. Soc. exp. Biol. (N.Y.) 107, 491-494 (1961).

7. Hazzard, W. R., Crockford, P. M., Buchanan, K. D., Vance, J.E., Chen, R., Williams, R. H.: A double antibody immunoassay for glucagon. Diabetes 17, 179186 (1968).

8. Heding, L.G.: The production of glucagon antibodies in rabbits. Horm. Metab. Res. 1, 87-88 (1969).

9. Herbert, V., Lau, K.S., Gottlieb, C.W., Bleicher, S.J.: Coated charcoal immunoassay of insulin. J. clin. Endocr. 25, 1375-1384 (1965).

10. Lawrence, A.M.: Radioimmunoassayable glucagon levels in man: effects of starvation, hypoglycemia, and glucose administration. Proc. nat. Acad. Sci. (Wash.) 55, 316-320 (1966).

11. Meyer, V., Knobil, E.: Growth hormone secretion in the unanesthetized rhesus mokey in response to no. xious stimuli. Endocrinology 80, 163-171 (1967).

12. Mirsky, A., Perisutti, G., Davis, N.C.: The destruction of glucagon by the blood plasma from various species. Endocrinology 64, 992-1001 (1959).

13. Nonaka, K., Foa, P.P.: A simplified glucagon immunoassay and its use in a study of incubated pancreatic islets. Proc. Soc. exp. Biol. (N.Y.) 130, 330$336(1969)$.

14. Rosselin, G., Tchobroutsky, G., Assan, R., Drouet, J., Dolais, J., Freychet, M., Dérot, M. : La méthode do dosage radio-immunologique d'étude des hormones protéiques de Berson et Yalow. Ann. Endocr. (Paris) 26, 449-464 (1965).

15. Samols, E., Marks, V.: Nouvelles conceptions sur la signification fonctionelle du glucagon (pancréatique et extrapancréatique). J. Diabét. Hôtel Dieu 1967, $43-66$ (1967). 
16. - Tyler, J., Megyesi, C., Marks, V.: Immunochemical glucagon in human pancreas, gut and plasma. Lancet 1966 II, $727-729$.

17. - - Mialhe, P.: In "Proceedings of the Third International Congress of Endocrinology", Mexico (1968) (in the press).

18. Schopman, W., Hackeng, W.H.L., Steendijk, C.: The purification of ${ }^{125} \mathrm{I}$-glucagon of high specivity for radioimmunochemical estimation of glucagon and a qualitative comparison of glucagon from different sources. Acta endocr. (Kbh.) 54, 527-540 (1967).

19. Snedecor, G.W.: Query $\mathrm{n}^{\circ} 92$. Biometrics $8,85-86$ (1952).

20. Unger, R.H., Eisentraut, A.M.: Glucagon. In: "Hormones in blood", Gray, C.H., Bucharach, A.L. (Eds.), Vol. 1, 2nd Edition, pp. 83-128. Now-York: Academic Press 1967.

21. - - Etudes récentes sur la physiologie du glucagon. J. Diabét. Hôtel Dieu 1967, 7-18 (1967).
22. - - MeCall, M.S., Keller, S., Lanz, H.C., Madison, L.L.: Glucagon antibodies and their use for immunoassay for glucagon. Proc. Soc. exp. Biol. (N.Y.) 102, $621-623$ (1959).

23. Weinges, K.F.: Glucagon. Monographie in der Reihe Biochemie und Klinik, Hrsgb. Weitzel, G., Zöllner, N. Stuttgart: Thieme 1968 .

24. Worobec, R., Locke, R., Hall, A., Ertl, R., Ernst, K., Deiniger, E.: Production of antibodies of high binding affinities in rabbits. Biochem. biophys. Res. Comm. 29, 406-412 (1967)

Dr. V. Leclercq-Meyer

Laboratoire de Médecine Expérimentale

Université Libre de Bruxelles

115, Boulevard de Waterloo

Bruxelles 1, Belgique 